\title{
Energy Spectrum for a Short-Range 1/r Singular Potential with a Non-Orbital Barrier Using the Asymptotic Iteration Method
}

\author{
Abdullah J. Sous ${ }^{1}$, Abdulaziz D. Alhaidari ${ }^{2}$ \\ ${ }^{1}$ Faculty of Technology and Applied Sciences, Al-Quds Open University, Tulkarm, Palestine \\ ${ }^{2}$ Saudi Center for Theoretical Physics, Jeddah, Saudi Arabia \\ Email: asous@qou.edu
}

Received 21 December 2015; accepted 19 January 2016; published 22 January 2016

Copyright (C) 2016 by authors and Scientific Research Publishing Inc.

This work is licensed under the Creative Commons Attribution International License (CC BY). http://creativecommons.org/licenses/by/4.0/

(c) (i) Open Access

\begin{abstract}
Using the asymptotic iteration method, we obtain the $S$-wave solution for a short-range three-parameter central potential with $1 / \mathrm{r}$ singularity and with a non-orbital barrier. To the best of our knowledge, this is the first attempt at calculating the energy spectrum for this potential, which was introduced by $\mathrm{H}$. Bahlouli and A. D. Alhaidari and for which they obtained the "potential parameter spectrum". Our results are also independently verified using a direct method of diagonalizing the Hamiltonian matrix in the J-matrix basis.
\end{abstract}

\section{Keywords}

Short-Range Three-Parameter Central Potential, Asymptotic Iteration Method, Potential Parameter Spectrum Method, J-Matrix, Diagonalizing Method, Energy Eigenvalues

\section{Introduction}

In previous work [1], we used the Asymptotic Iteration Method (AIM) to find the energy spectrum for the hyperbolic single wave potential, which was introduced by Bahlouli and Alhaidari [2] [3]. In the present work, we apply the same technique in [1] to the short-range three-parameter central potential, which was introduced by the same authors [3] [4] as

$$
V(r)=V_{0} \frac{\mathrm{e}^{-\lambda r}-\gamma}{\mathrm{e}^{\lambda r}-1}
$$

How to cite this paper: Sous, A.J. and Alhaidari, A.D. (2016) Energy Spectrum for a Short-Range 1/r Singular Potential with a Non-Orbital Barrier Using the Asymptotic Iteration Method. Journal of Applied Mathematics and Physics, 4, 79-85. 
where $V_{0}$ is the potential strength and the range parameter $\lambda$ is positive with an inverse length units. The dimensionless parameter $\gamma$ is in the open range $0<\gamma<1$. This potential is short-range with $1 / r$ singularity at the origin. It is also interesting to note that, at short distance and with $0<\gamma<1$, there is a clear resemblance of this potential with $V_{0}>0$ to the attractive Coulomb potential [5] with non-zero angular momentum (see Figure 1). However, the potential valley here is not due to the centrifugal force attributed to the angular momentum. Moreover, it does not have the long-range behavior of the Coulomb potential [3] [4]. In [4], Alhaidari argued that in contrast to the Coulomb potential the number of bound states for this potential is finite and that it could be used as a more appropriate model for the description of an electron interacting with an extended molecule whose electron cloud is congregated near the center of the molecule. The authors of [2]-[4] found the "potential parameter spectrum" (PPS) for the hyperbolic single wave potential and for potential (1). The concept of a PPS was introduced for the first time in the solution of the wave equation in [6] where for a given energy the problem becomes exactly solvable for a discrete set (finite or infinite) of values of the potential parameters. If the map that associates the parameter spectrum with the energy is invertible, then in principle one could obtain the energy spectrum for a given choice of potential parameters [6].

The calculation of energy eigenvalues is one of the basic problems of elementary quantum mechanics. Many techniques have been suggested to obtain the energy spectrum associated with a given potential. We will not try to give an overview here but one may consult [7]-[15] and references therein for the description of the AIM, which has been used successfully in the past. In this work, we will use this method to solve the time-independent S-wave (zero angular momentum) Schrödinger equation with potential (1) in order to find the energy spectrum.

The paper has the following structures. In Section 2, we briefly present an overview of the AIM and apply it to obtain the energy spectrum for potential (1). In Section 3, we find the eigen-energies where we make a comparison between the results obtained by the AIM and those obtained by the potential parameter spectrum method (PPSM). Additionally, we confirm our results independently by diagonalizing the Hamiltonian matrix, which is calculated in the J-matrix basis [16] [17].

\section{Basics of the AIM and Its Application to Potential (1)}

In this section, we present the basic concepts of the AIM. For more details, we refer the reader to [7]-[15]. The method could be used to solve a second-order homogeneous linear differential equations of the form

$$
y^{\prime \prime}(x)=k_{0}(x) y^{\prime}(x)+z_{0}(x) y(x)
$$

where $k_{0}(x)$ and $z_{0}(x)$ are continuously differentiable functions over the defined interval of the coordinate $x$ and the prime stands for the derivative with respect to $x$. According to the asymptotic aspect of the method and for sufficiently large $n$ we have

$$
\frac{z_{n}(x)}{k_{n}(x)}=\frac{z_{n-1}(x)}{k_{n-1}(x)}=\phi(x)
$$

where the function $\phi(x)$ is independent of $n$ and where

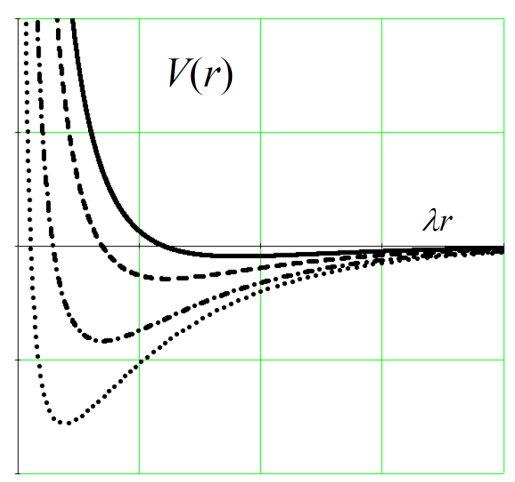

Figure 1. The potential function of Equation (1) for $\gamma=0.3$ (solid), $\gamma=0.5$ (dashed), $\gamma=0.75$ (dashed-dotted), $\gamma=0.9 \quad$ (dotted). 


$$
k_{n}(x)=k_{n-1}^{\prime}(x)+z_{n-1}(x)+k_{0}(x) k_{n-1}(x) \quad z_{n}(x)=z_{n-1}^{\prime}(x)+z_{0}(x) k_{n-1}(x)
$$

The general solution of Equation (2) is obtained as

$$
y(x)=\exp \left[-\int_{x} \phi(t) \mathrm{d} t\right]\left\{C_{2}+C_{1} \int_{x} \exp \left[\int_{t}\left(k_{0}(\tau)+2 \phi(\tau)\right) \mathrm{d} \tau\right] \mathrm{d} t\right\}
$$

The energy eigenvalues, $E$, of the Schrödinger equation corresponding to Equation (2) are obtained using the iteration terminating condition by means of Equation (3) that reads

$$
\Delta_{n}(x)=k_{n-1}(x) z_{n}(x)-z_{n-1}(x) k_{n}(x)
$$

If the problem is analytically solvable (exact solution) then $\Delta_{n}(x)=0$. In this case, the AIM gives the eigenvalues and eigenfunctions in explicit algebraic form. However, in this case there are a limited number of potentials and the condition $\Delta_{n}(x)=0$ is satisfied at every point $x$ in the defined interval. That is, $\Delta_{n}(x)$ is independent of $x$ and the eigenvalues are determined from the $n$ roots of the condition $\Delta_{n}(x)=0$. In case of approximation, $\Delta_{n}(x)$ depends on both $x$ and $E$. So, we have to determine a proper initial point $x=x_{0}$ value for solving $\Delta_{n}(x)=0$ with respect to $E$. Ideally, the solution should be independent of the choice of $x_{0}$. However, an acceptable range of stability of the calculated energy eigenvalue may be found near the minimum value of the potential or the maximum value of the asymptotic wave function [14] [15]. Next, we apply the method to the problem with the potential function (1).

Inserting potential (1) in the time-independent radial Schrödinger's equation results in the following second order linear differential equation

$$
\left[-\frac{1}{2} \frac{\mathrm{d}^{2}}{\mathrm{~d} r^{2}}+\frac{\ell(\ell+1)}{2 r^{2}}+V_{0} \frac{\mathrm{e}^{-\lambda r}-\gamma}{\mathrm{e}^{\lambda r}-1}-E\right] \psi(r)=0
$$

where $\ell$ is the angular momentum quantum number and we have adopted the atomic units $\hbar=m=1$. Defining the new variable $x=1-2 \mathrm{e}^{-\lambda r}$, whose range is between -1 and +1 , transforms this equation for $S$-wave $(\ell=0)$ into the desired Equation (2) where we can apply the AIM with

$$
\begin{gathered}
k_{0}(x)=\frac{1}{1-x} \\
z_{0}(x)=\frac{2 V_{0}}{\lambda^{2}}\left[\frac{1 / 2}{1+x}-\frac{\gamma}{1-x^{2}}-\frac{E / V_{0}}{(1-x)^{2}}\right]
\end{gathered}
$$

Using these seed functions together with the quantization condition (6) in the iteration, we can calculate the energy eigenvalues. Since the problem is not exactly solvable, we have to select a proper $x_{0}$. In this work, we observed that the best initial point is $x_{0}=0$, which corresponds to the middle of the $x$ interval. Therefore, at the end of the iterations when a stable result is reached, the energy spectrum is obtained by substituting $x=0$.

\section{Results and Discussion}

The calculation of the energy eigenvalues for potential (1) is not as easy as that for the hyperbolic single wave potential, which we have studied in [1]. This is because the present potential has $1 / r$ singularity at the origin. In this work, we take the value of the parameter $\gamma$ between zero and one. If $\gamma$ is less than zero or larger than one then the potential is not too interesting since it will not have the potential valley or potential hill (see Figure 1) and will only be similar to the well-known Hulthen or Yukawa potentials [18]. The necessary but not sufficient conditions for the existence of bound states for this potential are as follows:

1) If $\gamma$ is between zero and one, then any value of $V_{0}$ positive or negative could in principle support bound states.

2) If $\gamma$ is greater than one or less than zero, then the sign of $V_{0}$ must be the same as that of $\gamma$.

In Table 1, we compare the energy spectrum obtained using the AIM outlined above and those obtained by the PPSM for $V_{0}=5, \lambda=0.2$ and for several values of $\gamma$ between zero and one. The Table shows good agreement between the two results. However, the PPSM results are in better agreement with the direct method of diagonalization of the Hamiltonian matrix in the J-matrix basis (HDM). In the Appendix, we show how this diagonalization procedure is carried out. Table 2 shows also another good agreement between the results obtained 
Table 1. Energy spectrum with $V_{0}=5$ and $\lambda=0.2$ for various values of $\gamma$ obtained using the three methods mentioned in the text.

\begin{tabular}{ccccc}
\hline$n$ & method & $\gamma=0.2$ & $\gamma=0.4$ & $\gamma=0.6$ \\
\hline & AIM & -0.02600017100 & -0.1794066345 & -0.5368000468 \\
0 & PPSM & -0.0260054988 & -0.1794066345 & -0.5368000468 \\
& HDM & -0.0260054988 & -0.1794066345 & -0.31823436000468 \\
& AIM & -0.001138151383 & -0.07260826273 & -0.3182343338 \\
1 & PPSM & -0.0002851232 & -0.0726083684 & -0.3182343338 \\
& HDM & -0.0002791687 & -0.0726083684 & -0.1627941441 \\
& AIM & & -0.01483611943 & -0.1627941432 \\
2 & PPSM & & -0.0146815305 & -0.062326951432 \\
& HDM & & -0.0146815305 & -0.0623301374 \\
& AIM & & & -0.0623301374 \\
3 & PPSM & & & -0.01131548300 \\
& HDM & & & -0.0103706012 \\
& AIM & & & -0.0103705985 \\
\hline
\end{tabular}

Table 2. Energy spectrum with $\gamma=0.6$ and $\lambda=0.5$ for various values of $V_{0}$. The results of the three independent methods are in good agreement.

\begin{tabular}{ccccc}
\hline$n$ & method & $V_{0}=20$ & $V_{0}=40$ & $V_{0}=60$ \\
\hline \multirow{2}{*}{0} & AIM & -2.017967507 & -4.417015612 & -6.886516026 \\
& PPSM & -2.0179675071 & -4.4170156123 & -6.8865160257 \\
& HDM & -2.0179675071 & -4.4170156123 & -4.825915095 \\
1 & AIM & -1.008842615 & -2.815063004 & -4.8259150953 \\
& PPSM & -1.0088426139 & -2.8150630039 & -4.8259150953 \\
& HDM & -1.0088426139 & -2.8150630039 & -3.184708114 \\
2 & AIM & -0.3740938860 & -1.617682362 & -3.1847081143 \\
& PPSM & -0.3740996421 & -1.6176823617 & -1.1847081143 \\
& HDM & -0.3740996421 & -1.6176823617 & -1.920830979 \\
3 & AIM & -0.06098871935 & -0.7773062284 & -1.9208309792 \\
& PPSM & -0.0568940453 & -0.7773055229 & -0.9991295725 \\
& HDM & -0.0568940452 & -0.7773055229 & -0.9991291965 \\
4 & AIM & & -0.2548982339 & -0.9991291965 \\
& PPSM & & -0.2553793984 & -0.3895811680 \\
& HDM & & -0.2553793984 & -0.3897965892 \\
5 & AIM & & -0.04015268525 & -0.3897965892 \\
& PPSM & & -0.0200363806 & -0.08198118960 \\
& HDM & & -0.0200357906 & -0.0672459224 \\
& AIM & & & -0.0672459104 \\
& PPSM & & & \\
\hline
\end{tabular}

by the two methods but now $\gamma$ is fixed while $V_{0}$ is varied.

\section{Acknowledgements}

The second author (ADH) would like to dedicate his contribution in this work to his friend, teacher and mentor Prof. Hashim A. Yamani on the occasion of his 70th birthday. Without his continued support and counsel, I could not have utilized his formulation of the J-matrix theory to the advancement of the tridiagonal representation approach, which was instrumental in the solution of the current and many other problems. 


\section{References}

[1] Sous, A.J. (2015) The Asymptotic Iteration Method for the Eigen-Energies of the Novel Hyperbolic Single Wave Potential. Journal of Applied Mathematics and Physics, 3, 1406-1411.

[2] Bahlouli, H. and Alhaidari, A.D. (2010) Extending the Class of Solvable Potentials: III. The Hyperbolic Single Wave. Physica Scripta, 81, Article ID: 025008. http://dx.doi.org/10.1088/0031-8949/81/02/025008

[3] Alhaidari, A.D. and Bahlouli, H. (2009) Two New Solvable Potentials. Journal of Physics A: Mathematical and Theoretical, 42, Article ID: 262001. http://dx.doi.org/10.1088/1751-8113/42/26/262001

[4] Alhaidari, A.D. (2010) Extending the Class of Solvable Potentials: II. Screened Coulomb Potential with a Barrier. Physica Scripta, 81, Article ID: 025013. http://dx.doi.org/10.1088/0031-8949/81/02/025013

[5] Rogers, F.J., Graboske Jr., H.C. and Harwood, D.J. (1970) Bound Eigenstates of the Static Screened Coulomb Potential. Physical Review A, 1, 1577. http://dx.doi.org/10.1103/PhysRevA.1.1577

[6] Alhaidari, A.D. (2007) Representation Reduction and Solution Space Contraction in Quasi-Exactly Solvable Systems. Journal of Physics A: Mathematical and Theoretical, 40, 6305. http://dx.doi.org/10.1088/1751-8113/40/24/004

[7] Ciftci, H., Hall, R.L. and Saad, N. (2003) Asymptotic Iteration Method for Eigenvalue Problems. Journal of Physics A: Mathematical and Theoretical, 36, Article ID: 11807. http://dx.doi.org/10.1088/0305-4470/36/47/008

[8] Ciftci, H., Hall, R.L. and Saad, N. (2005) Construction of Exact Solutions to Eigenvalue Problems by the Asymptotic Iteration Method. Journal of Physics A: Mathematical and Theoretical, 38, 1147-1155. http://dx.doi.org/10.1088/0305-4470/38/5/015

[9] Saad, N., Hall, R.L. and Ciftci, H. (2006) Sextic Anharmonic Oscillators and Orthogonal Polynomials. Journal of Physics A: Mathematical and Theoretical, 39, 8477-8486. http://dx.doi.org/10.1088/0305-4470/39/26/014

[10] Ozer, O. and Roy, P. (2009) The Asymptotic Iteration Method Applied to Certain Quasinormal Modes and Non-hermitian Systems. Central European Journal of Physics, 7, 747-752.

[11] Sous, A.J. (2006) Exact Solutions for a Hamiltonian Potential with Two-Parameters Using the Asymptotic Iteration Method. Chinese Journal of Physics, 44, 167-171.

[12] Soylu, A., Bayrak, O. and Boztosun, I. (2007) An Approximate Solution of Dirac-Hulthén Problem with Pseudospin and Spin Symmetry for Any $\kappa$ State. Journal of Mathematical Physics, 48, Article ID: 082302. http://dx.doi.org/10.1063/1.2768436

[13] Barakat, T. (2005) The Asymptotic Iteration Method for the Eigenenergies of the Anharmonic Oscillator Potential V(x) $=\mathrm{Ax}^{2 \alpha}+\mathrm{Bx}^{2}$. Physics Letters A, 344, 411-417. http://dx.doi.org/10.1016/j.physleta.2005.06.081

[14] Sous, A.J. (2006) Solution for the Eigenenergies of the Sextic Anharmonic Oscillator Potential $V(x)=A_{6} x^{6}+A_{4} x^{4}+$ $\mathrm{A}_{2} \mathrm{x}^{2}$. Modern Physics Letters A, 21, 1675. http://dx.doi.org/10.1142/S0217732306019918

[15] Sous, A.J. and El-Kawni, M.I. (2009) General Eigenvalue Problems with Unbounded Potential from Below. International Journal of Modern Physics A, 24, 4169. http://dx.doi.org/10.1142/S0217751X09044280

[16] Alhaidari, A.D., Bahlouli, H. and Abdelmonem, M.S. (2008) Taming the Yukawa Potential Singularity: Improved Evaluation of Bound States and Resonance Energies. Journal of Physics A: Mathematical and Theoretical, 41, Article ID: 032001. http://dx.doi.org/10.1088/1751-8113/41/3/032001

[17] Munger, C.T. (2007) Ideal Basis Sets for the Dirac Coulomb Problem: Eigenvalue Bounds and Convergence Proofs. Journal of Mathematical Physics, 48, Article ID: 022301. http://dx.doi.org/10.1063/1.2435595

[18] Imbo, T., Pagnamenta, A. and Sukhatme, U. (1984) Bound States of the Yukawa Potential via the Shifted 1/N Expansion Technique. Physics Letters A, 105, 183-187. http://dx.doi.org/10.1016/0375-9601(84)90393-1

[19] Alhaidari, A.D., Yamani, H.A. and Abdelmonem, M.S. (2001) Relativistic J-Matrix Theory of Scattering. Physical Review A, 63, Article ID: 062708. http://dx.doi.org/10.1103/PhysRevA.63.062708 


\section{Appendix: Diagonalization of the Hamiltonian Matrix in the J-Matrix Basis}

The Hamiltonian of the problem is $H=T+V(r)$, where the kinetic energy operator is $T=-\frac{1}{2} \frac{\mathrm{d}^{2}}{\mathrm{~d} r^{2}}+\frac{\ell(\ell+1)}{2 r^{2}}$ and $V(r)$ is the potential in (1). Diagonalization of the matrix representation of this Hamiltonian in a given basis is difficult for two reasons. First, it's due to the singular behavior at the origin, which goes like $1 / r^{2}$ for $T$ and like $1 / r$ for $V$. Second, it's the long-range behavior of $T$, which dictates that we need to deal with infinite dimensional matrices. On the other hand, if we manage to transfer the $1 / r$ singularity from $V$ and add it to $T$ by writing $V(r)=\frac{Z}{r}+U(r)$, where $\frac{Z}{r}=\lim _{r \rightarrow 0} V(r)$, then $Z=\frac{V_{0}}{\lambda}(1-\gamma)$ and we can write $H=H_{0}+U(r)$ where the reference Hamiltonian $H_{0}=T+\frac{Z}{r}$. Consequently, the new potential $U(r)$ is regular everywhere (see Figure A1) and thus it could be approximated very well by its matrix elements in a finite subset of a square integrable basis. Then, what remains is to have a full account for the matrix representation of the reference Hamiltonian $H_{0}$. However, $H_{0}$ is just the Coulomb Hamiltonian, which is known to have an exact tridiagonal matrix representation in the square integrable Laguerre basis with the following elements [16] [17]

$$
\chi_{n}(r)=a_{n}(\mu r)^{\ell+1} \mathrm{e}^{-\mu r / 2} L_{n}^{2 \ell+1}(\mu r)
$$

where $L_{n}^{v}(z)$ is the associated Laguerre polynomial of order $n$ in $z$ and $\mu$ is a length scale parameter. The normalization constant is chosen as $a_{n}=\sqrt{\Gamma(n+1) / \Gamma(n+2 \ell+2)}$. This gives an infinite tridiagonal symmetric matrix representation for the reference Hamiltonian whose elements read as follows

$$
\left(H_{0}\right)_{n m}=\frac{\mu^{2}}{4}\left[\left(n+\ell+1+\frac{4 Z}{\mu}\right) \delta_{n, m}+\frac{1}{2} \sqrt{n(n+2 \ell+1)} \delta_{n, m+1}+\frac{1}{2} \sqrt{(n+1)(n+2 \ell+2)} \delta_{n, m-1}\right]
$$

As noted above, the potential $U(r)$ is easily accounted for by its matrix elements in a finite subset of the basis (A1). Increasing the size of this subset will improve the accuracy of the results. These matrix elements are obtained by evaluating the integral

$$
\begin{aligned}
U_{n m} & =\mu \int_{0}^{\infty} \chi_{n}(r) U(r) \chi_{m}(r) \mathrm{d} r \\
& =a_{n} a_{m} \int_{0}^{\infty} y^{2 \ell+1} \mathrm{e}^{-y} L_{n}^{2 \ell+1}(y) L_{m}^{2 \ell+1}(y)[y U(y / \mu)] \mathrm{d} y
\end{aligned}
$$

where $y=\mu r$. The evaluation of this integral is performed using the Gauss quadrature approximation scheme associated with the Laguerre polynomials [19], which gives

$$
U_{n m} \cong \sum_{k=0}^{N-1} \Lambda_{n k} \Lambda_{m k}\left[\omega_{k} U\left(\omega_{k} / \mu\right)\right]
$$

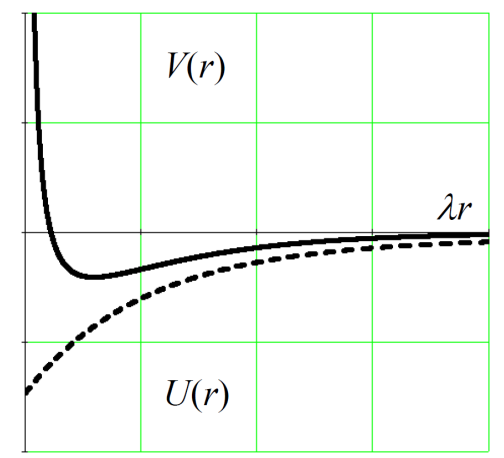

Figure A1. The potential function $V(r)$ in Equation (1) before removing the singularity (solid curve) and then after removing it as $U(r)$ (dashed curve). We took $\gamma=0.8$. 
for adequately large integer $N . \omega_{k}$ and $\left\{\Lambda_{n k}\right\}_{n=0}^{N-1}$ are the $N$ eigenvalues and corresponding normalized eigenvectors of the $N \times N$ tridiagonal basis overlap matrix whose elements are

$$
\left\langle\chi_{n} \mid \chi_{m}\right\rangle=2(n+\ell+1) \delta_{n, m}-\sqrt{n(n+2 \ell+1)} \delta_{n, m+1}-\sqrt{(n+1)(n+2 \ell+2)} \delta_{n, m-1}
$$

In an ideal situation where $N$ is infinite the physical results should be independent of the choice of value of the numerical scale parameter $\mu$. Nonetheless, for finite calculation we should be able to find a range of values of $\mu$ within which the results are stable and accurate to the desired number of significant digits. Increasing the size of the representation $N$ will increase this range, which we refer to as the plateau of stability. Thus, this parameter is analogous to the $x_{0}$ parameter in the AIM noted in Section 2 above. For a given set of physical parameters, we add (A2) to (A4) giving the matrix elements of the total Hamiltonian which could be diagonalized numerically for a proper value of the numerical scale parameter $\mu$ chosen from within the plateau of stability and for large enough matrix size $N$.

Table 1 and Table 2 show an excellent agreement of the results obtained by this diagonalization method (HDM) for $\ell=0$ with the PPSM and shows good to fair agreement with the AIM results. The agreement is less pronounced for the highest energy states. In the HDM, we used a basis size of $N=100$. 